\title{
Rupture des tiges en flexion
}

\author{
Basile Audoly a et SÉbastien Neukirch \\ Laboratoire de Modélisation en Mécanique, CNRS UMR 7607, Université Pierre et Marie Curie, 4 place Jussieu, \\ 75252 Paris Cedex 05, France
}

Reçu le 25 février 2005, accepté le 21 mars 2005

\begin{abstract}
Résumé - Lorsqu'on brise en flexion des tiges cassantes, telles que les spaghetti crus, on obtient plus de deux morceaux, souvent trois, quatre ou plus. Dans le but d'expliquer ces brisures multiples, nous étudions la dynamique d'une tige fléchie juste en-deçà de sa courbure de rupture lorsqu'elle est soudainement libérée à une extrémité. Nous trouvons que la relaxation brusque de la courbure au bout qui vient d'être libéré induit un train d'onde de flexion dont la dynamique est décrite par une solution auto-semblable sans paramètre ajustable. Ces ondes de flexion augmentent localement la courbure et nous montrons que ce mécanisme contre-intuitif est à l'origine de la fragmentation des tiges cassantes en flexion. Nous présentons une expérience simple qui confirme cette assertion.
\end{abstract}

Mots clés : Équations de Kirchhoff dynamiques / rupture fragile

\begin{abstract}
How bent rods break. When thin brittle rods such as dry spaghetti pasta are bent beyond their limit curvature, they often break into more than two pieces, typically three or four. With the aim to understand these multiple breakings, we study the dynamics of a rod bent just below its limit curvature and suddenly released at one end. We find that the sudden relaxation of the curvature at the newly freed end leads to a burst of flexural waves, whose dynamics are described by a self-similar solution with no adjustable parameter. These flexural waves locally increase the curvature in the rod and we argue that this counter-intuitive mechanism is responsible for the fragmentation of brittle rods under bending. A simple experiment supporting the claim is presented.
\end{abstract}

Key words: Dynamic Kirchhoff equations / fragile fracture theory

La fragmentation concerne de nombreux domaines scientifiques et techniques. Plusieurs phénomènes physiques sont à l'œuvre, ce qui explique sans doute pourquoi la fragmentation a surtout été étudiée du point de vue statistique [1-5]. Des études récentes sont néanmoins fondées sur des considérations mécaniques ou physiques: contribution de l'énergie de surface [6], propriété de croissance et nucléation en théorie de la rupture [7], flambage dynamique [8,9] et propagation des ondes élastiques [10].

On considère une tige élastique tenue à ses deux extrémités, qui est progressivement courbée uniformément et de manière quasi-statique. Elle se casse au temps $t=0$ lorsque sa courbure $\kappa_{0}$ atteint sa valeur limite $\kappa^{*}$ : une fissure dynamique se propage alors transversalement et casse la tige en deux morceaux. La courbure avant rupture étant uniforme, la position du premier point de rupture est sélectionnée par les défauts. On ne discutera pas plus en détail cette rupture initiale pour se

a Auteur correspondant : k91d@lmm.jussieu.fr concentrer sur ce qui s'ensuit : nous étudions l'évolution dynamique de chacun des deux fragments ainsi créés pour $t>0$, et montrons qu'elle conduit à des nouvelles ruptures à des temps ultérieurs.

Comme on ne s'intéresse pas à la première rupture, on introduit un modèle simplifié de catapulte, que l'on étudie dans toute la suite : la libération d'une tige fléchie sert à simuler la première rupture. On se débarrasse ainsi de l'incertitude sur la position de la première rupture, la longueur $L$ de la catapulte étant connue par avance. Les deux problèmes (l'évolution de chacun des deux fragments juste après la première rupture d'une tige en flexion d'une part et la libération soudaine d'une tige à une extrémité d'autre part) obéissent aux mêmes équations. Dans notre problème de tige libérée, que nous désignerons par le terme de catapulte, la tige est initialement courbée et au repos. On obtient cet état initial en encastrant une extrémité et en appliquant sur l'autre extrémité un moment fléchissant $M_{0}$, que l'on fait disparaître au temps initial $t=0$ en libérant soudainement la tige d'un seul 


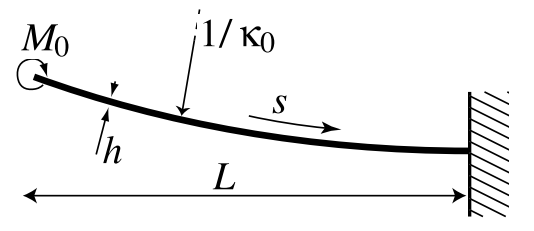

Fig. 1. L'évolution dynamique d'un fragment qui suit la rupture initiale d'une tige fragile est modélisée par la libération au temps $t=0$ d'une tige de longueur $L$ fixée, de courbure initiale $\kappa_{0}$ ne possédant pas de vitesse initiale.

côté. La tige n'est alors plus en équilibre et on étudie son évolution dynamique aux temps ultérieurs.

La dynamique des tiges est décrite par les équations de Kirchhoff [11] qui dans la limite des faibles fléchissements s'écrivent :

$$
L^{4} \kappa_{, s^{4}}(s, t)+T^{2} \kappa_{, t^{2}}(s, t)=0,
$$

où les virgules en indices dénotent les dérivées partielles. Nous avons introduit un temps caractéristique $T$ construit à partir des propriétés mécaniques de la tige : $T=L^{2} / \gamma$ où $\gamma=\sqrt{E I /(\rho A)}$, avec $E$ le module d'Young, $\rho$ la masse volumique, $A$ l'aire et $I$ le moment principal d'inertie de la section. Pour une tige de section circulaire de rayon $r$, $I=\pi r^{4} / 4$ et $\gamma=c r / 2$, où $c=\sqrt{E / \rho}$ désigne la vitesse du son dans le matériau. Notons que $T$ est directement proportionnel à la période du fondamental pour les petites oscillations libres de la tige, $T_{\text {free }}=1,79 \mathrm{~T}$.

Dans l'équation (1), on cherche à déterminer l'inconnue $\kappa(s, t)$, fonction de l'abscisse curviligne $s$ et du temps $t$. On utilise les équations linéarisées pour les petites oscillations dans le seul but de simplifier l'exposé : nous avons effectué des calculs numériques fondés sur les équations complètes non-linéaires de Kirchhoff, et n'avons pas obtenu de différence essentielle avec ce que la théorie linéaire permet de prédire. Les conditions initiales pour l'équation (1) sont des conditions d'encastrement en $s=L: \kappa_{, s^{2}}(L, t)=0, \kappa_{, s^{3}}(L, t)=0$, et des conditions de bord libre en $s=0: \kappa(0, t)=0, \kappa_{, s}(0, t)=0$. Avec les conditions initiales $\kappa(s, 0)=\kappa_{0}$ and $\kappa, t(s, 0)=0$ (courbure uniforme $\kappa_{0}$, pas de vitesse initiale), on s'attend en principe à une solution unique par intégration de l'équation aux dérivées partielles en temps et espace.

Il n'en est rien. Une analyse de ces conditions initiales et aux bords révèle la présence d'une incompatibilité : le problème est mathématiquement mal posé. Il se développe par conséquent une couche limite aux temps très courts $t \sim T_{s} \sim r / c$ : la courbure au bord qui vient d'être libéré est initialement non nulle mais relaxe extrêmement rapidement vers zéro. Durant cette phase très rapide, les approximations faites pour établir l'équation (1) sont trop grossières. La conséquence [12] est que, pour décrire les temps grands devant ce temps très court de relaxation (régime dit intermédiaire), il faut chercher une solution auto-semblable aux équations de Kirchhoff. Dans ce régime intermédiaire :

$$
T_{s} \ll t \ll T
$$
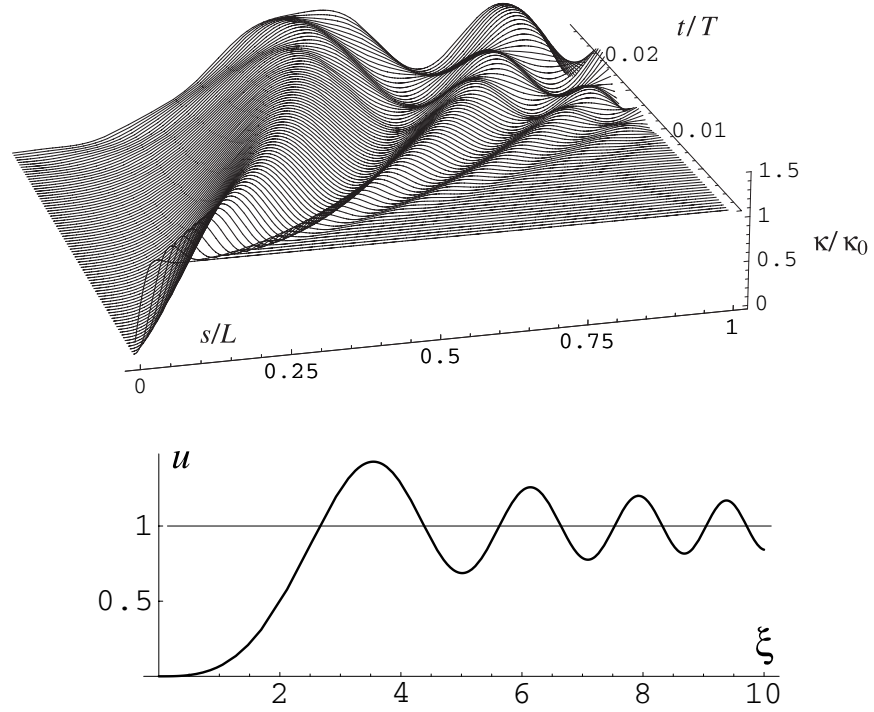

Fig. 2. En haut : simulation numérique des équations de Kirchhoff dynamiques pour une configuration initiale en demicercle $\kappa_{0}=\pi / L$. La courbure au bord libéré $\kappa(0, t)$ relaxe vers zéro en quelques pas de temps puis est donnée dans le régime intermédiaire (2) par la solution auto-semblable (3). Aux temps ultérieurs, des réflections se produisent sur le bord encastré $s=L$. En bas : solution $u=\kappa / \kappa_{0}$ auto-semblable décrivant le régime intermédiaire $\xi=s / \sqrt{\gamma t}$.

l'évolution de la tige est décrite par une solution analytique auto-semblable :

$$
\kappa(s, t)=2 \kappa_{0} S\left(\frac{1}{\sqrt{2 \pi}} \frac{s}{\sqrt{\gamma t}}\right),
$$

où $S(x)=\int_{0}^{x} \sin \left(\frac{\pi}{2} y^{2}\right)$ d $y$ est l'intégrale sinus de Fresnel qui apparaît en théorie de la diffraction. L'équation (3) ne décrit pas une solution progressive classique, $s \sim c t$, mais plutôt une solution auto-semblable $s \sim \sqrt{\gamma t}$. Ceci est relié aux propriétés dispersives de l'équation d'ondes (1).

Toutes les tiges qui sont libérées soudainement à un bord sont décrites par la même solution autosemblable (3) dans le régime intermédiaire (2), quelles que soient les propriétés du matériau, les détails de la libération initiale (qui doit néanmoins se produire sur un temps très court $T_{s} \ll T$ ), et même les conditions initiales imposées sur le bord opposé. Cette solution universelle est tracée sur la figure 2 (partie basse), et comparée à une simulation numérique des équations de Kirchhoff (Fig. 2, partie haute). La solution numérique révèle bien, comme attendu, un régime auto-semblable pour $T_{s} \ll$ $t \ll T$, pendant lequel un train d'onde de flexion parcourt la tige depuis le bord libéré $s=0$ jusqu'au bord encastré, la longueur parcourue augmentant avec la racine carrée du temps. La solution auto-semblable (3) décrit bien l'évolution dynamique de la tige jusqu'à ce que des réflections se produisent sur le bord encastré $s=L$, pour un temps $t \sim T$. Pour décrire ces réflexions, il faudrait alors combiner la solution auto-semblable (3) avec ses réflections obtenues par la méthode des images. 
Une propriété essentielle de la solution auto-semblable est qu'elle prédit localement un accroissement de courbure par rapport à la courbure initiale $\kappa_{0}$. L'étude du maximum de l'intégrale sinus de Fresnel montre qu'un maximum de courbure se produit dans le régime intermédiaire pour $(s / L) / \sqrt{t / T}=2 \sqrt{\pi}$. La valeur de ce maximum de courbure rapportée à la courbure initiale est un nombre universel, $\kappa_{\mathrm{m}} / \kappa_{0}=1,428$. Juste après la libération du bord libre, et jusqu'à ce que des réflexions se produisent, un train d'onde de courbure traverse la tige de part en part, augmentant localement la courbure de $42,8 \%$. Ce résultat est assez contre-intuitif, car on s'attendrait plutôt, en libérant le bord libre, à diminuer les déformations dans la tige. Il est vrai que sur le long terme, la tige va retourner à un état rectiligne, mais nous venons de voir grâce à la solution auto-semblable que sa dynamique très particulière aux temps courts induit une augmentation locale de la courbure.

Si l'on se souvient que la tige possède une courbure initiale $\kappa_{0}$ qui coïncide avec sa courbure limite de rupture $\kappa^{*}$, on voit que l'augmentation de courbure qui suit la libération d'un bord de la tige est incompatible avec le critère de stabilité à la rupture en flexion, $\kappa<\kappa^{*}$. On en déduit que, si l'état initial est suffisamment chargé, la tige peut rompre uniquement parce qu'elle est relâchée. Autrement dit, une catapulte sans charge embarquée peut rompre pour la seule raison qu'elle est déclenchée. Cette assertion plutôt surprenante peut être vérifiée par une expérience relativement simple, voir la figure 3 . On courbe un spaghetti en arc de cercle jusqu'à une courbure proche de sa courbure limite. On le relâche brusquement à un bord. On observe alors souvent que le spaghetti rompt très rapidement, en un endroit plus ou moins éloigné du point de libération, parfois même près du bord opposé. En filmant cette expérience à la caméra rapide, nous avons confirmé le rôle du train d'ondes de flexion décrit par l'équation (3). La figure 3 montre des clichés expérimentaux superposés avec le calcul numérique sans paramètre ajustable. La rupture se produit exactement au point calculé de courbure maximale. En comparant le rayon de courbure initial sur cette figure, et les rayons de courbure beaucoup plus faibles atteints durant l'évolution dynamique de la tige, on voit qu'en effet, et contrairement à l'intuition, la courbure de la tige augmente bien localement.

Nous avons donc expliqué comment une tige fragile casse lorsqu'elle est libérée à la façon d'une catapulte. Or cette expérience de la catapulte avait précisément été introduite parce qu'elle est régie par les mêmes équations qu'une moitié de tige en flexion venant de subir sa première rupture. On en déduit donc qu'une tige en flexion qui rompt à un instant donné subit des cassures secondaires presque immédiatement après la rupture initiale. Pour étudier les ruptures suivantes dans une tige en flexion, il faudrait étudier la dynamique des sous-fragments. La difficulté est alors qu'on ne part pas d'une solution de courbure constante, mais d'une condition initiale plus complexe pour le profil de courbure. Les courbures postérieures sont donc plus difficiles à étudier,

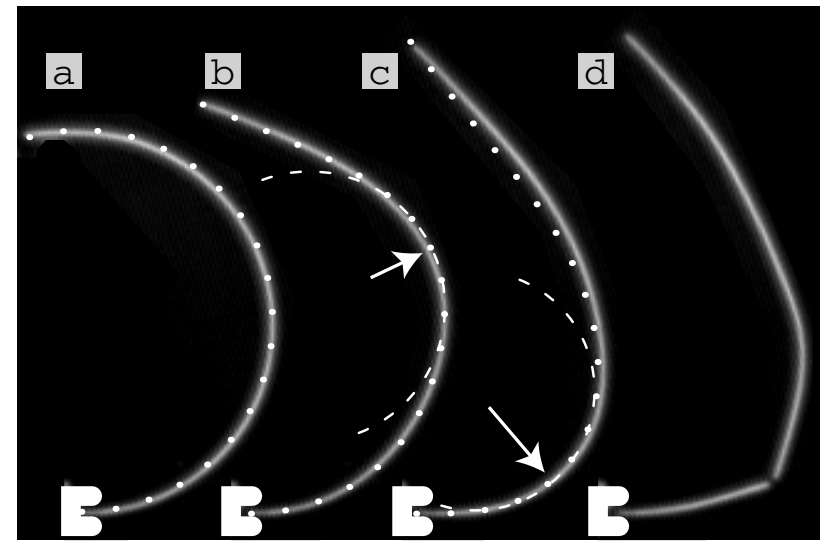

Fig. 3. Un spaghetti peut se casser quand on libère simplement une de ses extrémités. La pâte est d'abord fléchie en arc de cercle jusqu'à une courbure légèrement inférieure à sa courbure de rupture. L'extrémité inférieure est encastrée. L'extrémité supérieure est libérée soudainement, au temps $t_{\mathrm{a}}=0$. On montre quelques images obtenues à la caméra rapide à $1000 \mathrm{~Hz}$ : (a) libération $t_{\mathrm{a}}=0$, (b) cliché intermédiaire $t_{\mathrm{b}}=0,0159 T$, (c) cliché immédiatement antérieur à la rupture $t_{\mathrm{c}}=0,0509 \mathrm{~T}$, et (d) immédiatement postérieur $t_{\mathrm{d}}=0,0596 \mathrm{~T}$. Les simulations numériques fondées sur les équations dynamiques de Kirchhoff sont superposées, sans paramètre ajustable : profil de la tige (pointillés), et cercle osculateur (tirets) au point de courbure maximale (flèche). Noter que la rupture se produit bien au point de courbure maximale.

mais pour une raison purement technique. Le mécanisme que nous avons mis en lumière n'en reste pas moins valable : toute rupture se traduit par la libération brusque de deux nouveaux fragments. Chacun de ces deux sousfragments suit une évolution dynamique semblable à celle que nous avons étudiée, caractérisée notamment par une augmentation locale de la courbure. Cette augmentation locale produit elle-même de nouveaux évènements de ruptures puisque la courbure initiale, proche de la courbure de rupture, est largement dépassée. On en déduit donc génériquement un mécanisme de rupture en cascade pour les tiges fragiles en flexion. Les seuls éléments susceptibles de limiter la taille finale des fragments sont liés à la dissipation de l'énergie (comportement plastique ou visco-élastique de la tige, dissipation de l'énergie durant la propagation d'une fissure transverse, etc.) ou à des phénomènes des retards à la rupture (la tige ne casse pas instantanément quand la courbure $\kappa$ atteint sa valeur limite $\kappa^{*}$ ).

L'étude de notre modèle de tige simple nous a permis de comprendre pourquoi les tiges fragiles ne cassent pas en général en deux morceaux. Le problème qu'il reste maintenant à comprendre est de savoir ce qui limite en pratique la cascade de rupture vers les petites échelles, et ce qui fixe la statistique des fragments ainsi créés.

\section{Références}

[1] N.F. Mott, E.H. Linfoot, Ministry of Supply Report No. AC3348, January 1943, unpublished 
[2] D.E. Grady, M.E. Kipp, Geometric statistics and dynamic fragmentation, J. Appl. Phys. 58 (1985) $1210-1222$

[3] R. Englman, Fragments of matter from a maximumentropy viewpoint, J. Phys: Condens. Matter 3 (1991) 1019-1053

[4] L. Oddershede, P. Dimon, J. Bohr, Self-organized criticality in fragmenting, Phys. Rev. Lett. 71(19) (1993) 3107-3110

[5] E.S.C. Ching, S.L. Lui, Ke-Qing Xia, Energy dependence of impact fragmentation of long glass rods, Physica A 287 (2000) 89-90

[6] L. Griffith, Can. J. Res. 21 (1943) 57-64

[7] N.F. Mott, Fragmentation of Shell Cases, Proc. Roy. Soc. London A 189 (1947) 300-308
[8] J.R. Gladden, N.Z. Handzy, A. Belmonte, E. Villermaux, Dynamic buckling and fragmentation in brittle rods, Phys. Rev. Lett. (2005, in press)

[9] F. Wittel, F. Kun, H.J. Herrmann, B.H. Kröplin, Fragmentation of shells, Phys. Rev. Lett. 93035504 (2004)

[10] D.A. Shockey, D.R. Curran, L. Seaman, J.T. Rosenberg, C.F. Peterson, Int. J. Rock. Mech. Min. Sci. 11 (1974) 303-317

[11] B.D. Coleman, E.H. Dill, M. Lembo, Z. Lu, I. Tobias, On the dynamics of rods in the theory of Kirchhoff and Clebsch, Arch. Rational Mech. Anal. 121 (1993) 339-359

[12] B. Audoly, S. Neukirch, Fragmentation of rods by cascading cracks: why spaghetti do not break in half, Phys. Rev. Lett. (2005, submitted) 